\title{
Is the contralateral lesser trochanter a reliable reference for planning of total hip arthroplasty - a 3-dimensional analysis
}

\author{
Julian Hasler ${ }^{1 *}$, Armando Hoch', Philipp Fürnstahl' ${ }^{2}$, Jakob Ackermann ${ }^{1}$, Patrick O. Zingg ${ }^{1}$ and \\ Lazaros Vlachopoulos ${ }^{1}$
}

\begin{abstract}
Background: Preoperative templating in total hip arthroplasty (THA) is mandatory to achieve appropriate offset and leg length equality. However, templating methods using the contralateral hip might be susceptible to errors resulting from side-differences in the femoral morphology. The distance of the lesser trochanter to the femoral head center (LTFHD) is a frequently used reference parameter for preoperative planning and intraoperative validation during THA. However, currently no three-dimensional (3D) analysis of side differences of the LTFHD exists.

Methods: Using Computer tomography (CT)-based surface models from 100 paired femora (50 cadavers), side-toside asymmetry of the LTFHD, femoral length, femoral head diameter (FHD) and femoral antetorsion were analyzed. Univariate linear regression models were established to evaluate potential associations between sides regarding LTFHD and FHD as well as a correlation of these parameters with each other.

Results: Statistically significant side-differences were found for the LTFHD $(p=0.02)$ and FHD $(p=0.03)$ with a mean absolute side-difference of $1.6 \pm 1.4 \mathrm{~mm}$ (range $0.1-5.5 \mathrm{~mm}$ ) and $0.4 \mathrm{~mm} \pm 0.6 \mathrm{~mm}$ (range $0-3 \mathrm{~mm}$ ), respectively. The ratio between the LTFHD and FHD was consistent with an average value of $1.16 \pm 0.08$ and reliable between sides with a correlation coefficient $(r)$ of $0.72(p<0.01)$.

Conclusions: The LTFHD is a reliable reference parameter for preoperative templating and intraoperative validation during THA with a high correlation between sides $(r=0.93, p<0.01)$. However, $8 \%$ of the investigated specimens revealed a LTFHD of more than $4 \mathrm{~mm}$, which should be anticipated during THA to avoid unsatisfiable results.
\end{abstract}

Keywords: 3-Dimensional analysis, Lesser trochanter, Center of femoral head, Distance, Side-to-side difference, Total hip arthroplasty

\section{Introduction}

Restoration of the native biomechanical setting in total hip replacements is crucial for achieving complete functional recovery, stability and good clinical outcomes $[1,2]$. To attain appropriate offset and limb-length equality, preoperative templating of total hip arthroplasty (THA) is

\footnotetext{
* Correspondence: julianhasler01@gmail.com

'Department of Orthopaedics, Balgrist University Hospital, University of Zurich, Forchstrasse 340, CH-8008 Zurich, Switzerland

Full list of author information is available at the end of the article
}

mandatory [3-7]. The distance between the lesser trochanter and the center of the femoral head (LTFHD) is a frequently used reference for preoperative planning and intraoperative validation to restore normal anatomy of the proximal femur and the hip [8-10]. However, due to altered anatomy and therefore distorted radiological representation of the proximal femur in severely arthritic hips, femoral neck fractures or collapse of the femoral head arising from osteonecrosis of the hip, the preoperative radiographic assessment of limp length and offset of the

(C) The Author(s). 2021 Open Access This article is licensed under a Creative Commons Attribution 4.0 International License, which permits use, sharing, adaptation, distribution and reproduction in any medium or format, as long as you give appropriate credit to the original author(s) and the source, provide a link to the Creative Commons licence, and indicate if changes were made. The images or other third party material in this article are included in the article's Creative Commons licence, unless indicated otherwise in a credit line to the material. If material is not included in the article's Creative Commons licence and your intended use is not permitted by statutory regulation or exceeds the permitted use, you will need to obtain permission directly from the copyright holder. To view a copy of this licence, visit http://creativecommons.org/licenses/by/4.0/ The Creative Commons Public Domain Dedication waiver (http://creativecommons.org/publicdomain/zero/1.0/) applies to the data made available in this article, unless otherwise stated in a credit line to the data. 
affected side might be of limited value [11].Thus, the unaffected hip is often used to plan and evaluate accuracy of hip reconstruction $[4,12,13]$. Furthermore, some authors propose the femoral head diameter as a valuable tool to predict the LTFHD [14-16], which might be helpful if pathological conditions exist on both hips. However, these templating methods based on plane radiographs may be susceptible to errors resulting from projection errors [17, 18] and side-to-side differences of the 3-dimensional geometry of the proximal femur [19-21].

While it is common practice to utilize the contralateral side for THA planning, only limited data exists regarding side-to-side variability of the LTFHD [14]. The current study, therefore, sought to establish a 3D analysis of the side-to-side difference of the LTFHD among individuals with normal anatomy of the proximal femur on full body CT. Understanding of normal variations of this commonly used parameter might improve the accuracy of preoperative planning in THA.

\section{Materials and Methods}

This study was approved by the institutional review board and the ethical committee (ID 2018-02242). CT full-body data of 50 cadaveric specimens provided by the Institute of Forensic Medicine, University of Zürich, Switzerland were included. There were 35 male and 15 female donors with an average age of $57 \pm 20$ years (median 58 years; range $18-86$ years). The average weight was $71 \pm 16 \mathrm{~kg}$ (median: $68 \mathrm{~kg}$; range: 41 to $110 \mathrm{~kg}$ ), the average height $171.5 \pm 15.9 \mathrm{~cm}$ (median: $172 \mathrm{~cm}$; range 137 to $190 \mathrm{~cm}$ ) and the average BMI was $24.1 \pm 5.2 \mathrm{~kg} /$ $\mathrm{m}^{2}$ (median: $22.7 \mathrm{~kg} / \mathrm{m}^{2}$; range: 17.3 to $44.8 \mathrm{~kg} / \mathrm{m}^{2}$ ). Exclusion criteria comprised any macroscopic pathology of the proximal femur visible on preoperative CT scan such as sequelae of childhood hip disorders including LeggCalvé-Perthes disease or slipped capital femoral epiphysis, malunion or femoral head collapse arising from osteonecrosis of the hip, as well as advanced degenerative changes of the hip.

\section{Generation of 3D triangular surface models}

CT full-body data of the included 50 cadaveric specimens were used to create $3 \mathrm{D}$ triangular surface models of 100 paired femora (50 right, 50 left). CT data were acquired using a Somatom Definition Flash CT scanner (Siemens Helathineers, Erlangen, Germany) with slice thickness of $1 \mathrm{~mm}$. In order to create 3D triangular surface models, segmentation of the femora was conducted using the global thresholding and region growing functionality of a standard segmentation software (Mimics Medical, Materialise NV, Leuven, Belgium). For further analysis, the generated surface models were imported into the planning software CASPA (Computer Assisted Surgery Planning Application), which was developed at
Balgrist University Hospital (Balgrist CARD [Computer Assisted Research and Development]).

\section{Definition of anatomical landmarks and parameters}

Defining the following landmarks was warranted to quantify the femoral anatomy. First, we calculated an oriented bounding box of the whole femoral model per principal component analysis [22], thereby deriving a local coordinate system as described by Jud et al [23]. The length of the longest side of the oriented bounding box was defined as the femoral length.

For the quantification of the femoral antetorsion, we defined the perpendicular axis of the posterior condyles (PPC) and the true femoral neck axis (FNA) [24].

For this purpose, the oriented bounding box was rotated around the $\mathrm{z}$-axis of this coordinate system and adjusted in its anteroposterior expansion until the most posterior points on the medial and lateral femoral condyles were identified. The axis connecting these points yields the PPC (Fig. 1 A). For definition of the true femoral neck axis (FNA), the femoral head center (FHC) was defined as the center of a best-fit sphere calculated form all surface points of the femoral head. Thereafter, a cylinder was fitted into the femoral neck with the FHC as center of the cylinder. The axis of the cylinder is called hereafter preliminary neck axis (PNA). A plane -

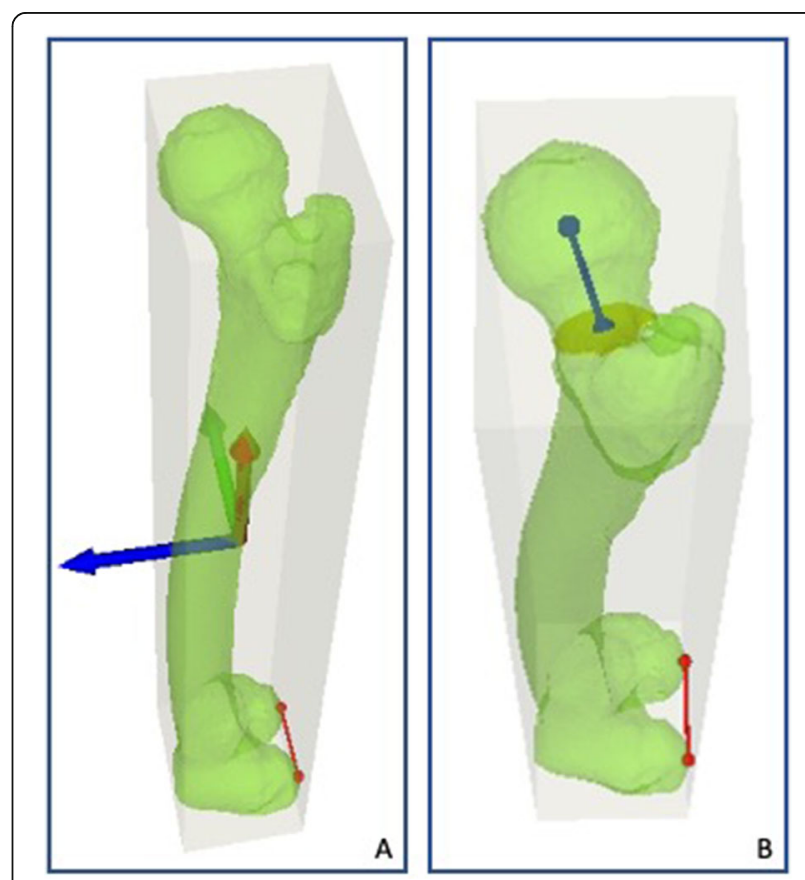

Fig. 1 Measurement of femoral antetorsion. a Red line: Perpendicular axis of the posterior condyles (PPC). b Blue line: Femoral neck axis (FNA), defined as the axis between the femoral neck center (FNC) and the femoral head center (FHC). Femoral antetorsion was defined as the angle between the PPC and the FNA, both projected on the xy-plane of the coordinate system 
preliminary neck plane (PNP) - with the PNA as plane normal was defined with a distance of 1.25 times the femoral head radius from the FHC. The intersection of the femoral model and the PNP was used to calculate the femoral neck center (FNC), as the center of mass of these points. The FNA, was defined as the axis between the FNC and the FHC. Femoral antetorsion was then defined as the angle between the PPC and the FNA, both projected on the xy-plane of the coordinate system. (Fig. 1B)

The LTFHD was measured as follows:

1. The femoral head center (FHC) was defined as the center of a best-fit sphere calculated form all surface points of the femoral head. The femoral head diameter was defined as the diameter of the sphere (FHD). (Fig. 2a)

2. Thereafter, a sphere with a diameter of $20 \mathrm{~mm}$ was placed in the center of the lesser trochanter (LTC). (Fig. 2a)

3. An axis connecting the LTC and the FHC was defined (LTFHA). A plane with the LTFHA as plane normal was placed on the upper end of the sphere fitted in the LTC. The intersection between this plane and the 3-D surface model of the femur was generated. (Fig. 2b)

4. The point at the upper border of the lesser trochanter (UBLT) was then defined as the point at the border of the previously generated intersection with the smallest distance to the center of the line connecting the LTC and the FHC. (Fig. 2c)
5. The distance between the UBLT and the FHC was defined as the LTFHD. (Fig. 2c)

Due to manual digitization involvement for the determination of the PPC, FNA, FHC, the FHD and the LTC, all measurements were performed by two independent blinded observers (J.H. and A.H.) for evaluation of interobserver reliabilities of the measurements.

\section{Statistical analysis}

Continuous variables are reported as mean and standard deviation (SD). Inter-reader reliabilities of the measurements were evaluated with the interclass correlation coefficients (ICC) with a 2-way random-effects model for absolute agreement. Normality of distribution was tested using the Shapiro-Wilk test. Accordingly, the two-tailed paired $\mathrm{t}$-test or Wilcoxon signed-rank test was applied to assess side-to-side differences based on the delta of the left minus right femur. Further, to assess a potential correlation between the FHD and the LTFHD, the ratio between the LTFHD and the FHD of the corresponding sides were calculated. Univariate linear regression models were established, to measure a possible two-way linear association between side-to-side differences of the geometry of the proximal femur. Statistical analysis was performed using SPSS Statistics (SPSS, IBM Corporation, 1 New Orchard Road Armonk, New York 10,504 - 1722, USA). P-values > 0.05 were considered statistically significant.

\section{Results}

Interobserver ICC ranged from 0.89 to 0.98 . The interobserver ICC was 1 for the femoral leg length (Table 1).
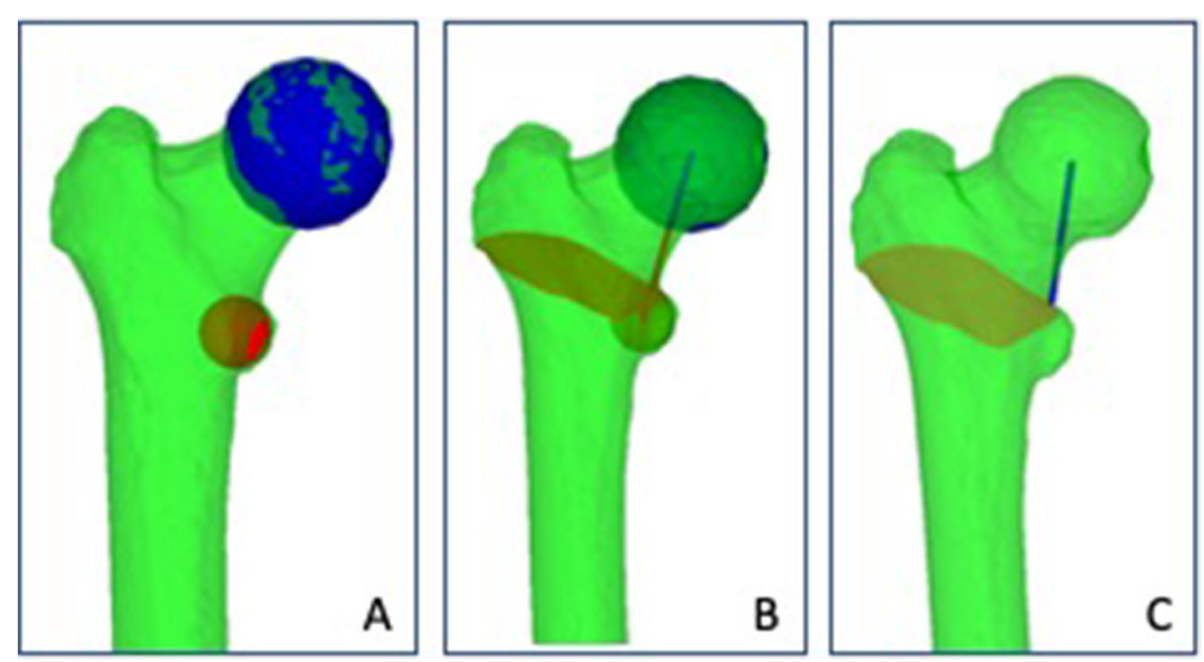

Fig. 2 Measurement of the LTFHD. a Blue sphere: FHC; Red Sphere: LTC. $\mathbf{b}$ Red plane: Intersection of the 3D surface model of the femur and a plane with the LTFHA (defined as the axis connecting the LTC and the FHC) as plane normal. c UBLT was defined as the point on the border of this intersection with the smallest distance to the line connecting the FHC and the LTC. Blue line connecting the UBLT and the FHC equals the LTFHD 
Table 1 Summary of Side-to-Side Differences in paired femora. SD, Standard deviation; FHD, femoral head diameter; LTFHD, lesser trochanter femoral head distance. ${ }^{*}$ indicates statistically significance difference $(p<0.05)$

\begin{tabular}{lccclcc}
\hline & $\begin{array}{l}\text { Total }(n=100) \\
\text { Mean (SD) }\end{array}$ & $\begin{array}{l}\text { Left }(n=50) \\
\text { Mean (SD) }\end{array}$ & $\begin{array}{l}\text { Right }(n=50) \\
\text { Mean (SD) }\end{array}$ & $\begin{array}{l}\text { Inter-observer interclass } \\
\text { correlation }\end{array}$ & $\begin{array}{l}\text { Absolute } \\
\text { Difference } \\
\text { Mean (range) }\end{array}$ & $\begin{array}{l}P \text {-value of } \\
\text { Side- } \\
\text { Difference }\end{array}$ \\
\hline $\begin{array}{l}\text { Femoral length }(\mathrm{mm}) \\
\text { Femoral Antetorsion }\end{array}$ & $466.0(29.3)$ & $466.3(29.9)$ & $465.7(28.9)$ & 1 & $3.4(01-14.9)$ & 0.36 \\
$\left(^{\circ}\right)$ & $10.0(7.6)$ & $10.7(7.4)$ & $9.4(7.8)$ & 0.93 & $4.0(0.1-13.0)$ & 0.06 \\
FHD $(\mathrm{mm})$ & $47.6(3.9)$ & $47.5(3.9)$ & $47.8(4.0)$ & 0.89 & $0.4(0-3)$ & $0.03^{*}$ \\
LTFHD $(\mathrm{mm})$ & $55.0(5.3)$ & $55.4(5.4)$ & $54.7(5.2)$ & 0.98 & $1.6(0.1-5.5)$ & $0.02^{*}$ \\
\hline
\end{tabular}

A statistically significant side-to-side-difference was found for the femoral head diameter $(p=0.03)$ and the LTFHD $(p=0.02)$ The mean absolute side-difference for LTFHD was $1.6 \pm 1.4 \mathrm{~mm}$ with a correlation coefficient (r) between sides of $0.93(p<0.01)$. Further information regarding side-to-side differences of the femoral anatomy are listed in Table 1 . In total, $70 \%$ of the paired femora showed an absolute side-difference in LTFHD $<$ $2 \mathrm{~mm}, 22 \%$ between 2 and $4 \mathrm{~mm}$, and $8 \%>4 \mathrm{~mm}$ (Table 2). Side-difference in LTFHD was not correlated to side-difference in femoral antetorsion $(r=0.07, p=$ $0.64)$ and femoral length $(r=0.4, p=0.74)$. Regarding the ratio of the FHD and the LTFHD, a mean ratio of $1.16 \pm 0.08$ with a correlation coefficient $(r)$ of $0.72(p<$ 0.01 ) was found between these parameters. A subgroup analysis comparing femora with an absolute sidedifference of more than $4 \mathrm{~mm}$ revealed no significant differences in patient demographics.

\section{Discussion}

The key finding of the current study was a statistically significant side-difference of the LTFHD $(p=0.02)$ with a mean absolute difference of $1.6+/-1.4 \mathrm{~mm}$ and a correlation coefficient $(r)$ between sides of $0.93(p<0.01)$. However, $8 \%$ of the specimens showed an absolute sidedifference of more than $4 \mathrm{~mm}$.

Side-to-side asymmetry of the femoral anatomy has been studied by various authors. Unnanuntana et al. used digital photographs of 200 cadaveric femora for a 2D analysis of different reference parameters frequently used to restore normal anatomy of the proximal femur during total hip replacement. In their study, they found an absolute side-difference of the LTFHD of $1.9 \pm$ $1.8 \mathrm{~mm}$ with a correlation coefficient between sides of

Table 2 Range of absolute difference in LTFHD and femoral length

\begin{tabular}{lll}
\hline & $\begin{array}{l}\text { Absolute Side-Difference } \\
\text { in LTFHD }\end{array}$ & $\begin{array}{l}\text { Absolute Side-Difference } \\
\text { in femoral length }\end{array}$ \\
\hline$<2 \mathrm{~mm}$ & $70 \%$ & $30 \%$ \\
$2-4 \mathrm{~mm}$ & $22 \%$ & $38 \%$ \\
$>4 \mathrm{~mm}$ & $8 \%$ & $32 \%$ \\
\hline
\end{tabular}

0.87 [14], which is in accordance with the results of the current study.

Other authors have attempted to develop alternative methods to predict the LTFHD than using measurements of the contralateral side. Polishchuk et al. retrospectively reviewed demographic and radiographic variables of 258 patients undergoing unilateral total hip arthroplasty or hemiarthroplasty. In their study, they found various variables including relative neck length, age, height, weight, gender and race to significantly correlate with LTFHD. Based on their findings, they derived an equation to predict LTFHD. However, although the average predicted LTFHD was within $2.9 \mathrm{~mm}$ of the intra-operatively measured values, the difference between the measured and predicted value varied up to $1.5 \mathrm{~cm}$, and the prediction only had a fair ICC of 0.65 [25]. Sproul et al. investigated a potential correlation of the FHD and the LTFHD. After examining the anatomy of 34 cadaveric femora, they concluded, that the LTFHD can be predicted by the following equation: FHD $(\mathrm{mm}) \mathrm{x}$ $1.035=$ LTFHD. However, their study entailed only a relatively small number of specimens and the correlation between the FHD and the LTFHD was only moderate $\left(r^{2}=0.46\right)$ [15]. Consistent with their findings, Unnanuntana et al. reported an average ratio between the LTFHD and the FHD of $1.01 \pm 0.12$ measured on plane photographs [14]. In our study, we found a slightly higher average ratio between the LTFHD and the FHD of 1.16 \pm 0.08 with a correlation coefficient $(r)$ between these parameters of 0.72 , which might be explained by the $3 \mathrm{D}$ measurement method of the FHD as well as the LTFHD. Taken together, these data suggest the FHD as an alternative option to predict the LTFHD, if measurement of the contralateral LTFHD is not possible due to distorted anatomy on both hips.

Atkinson et al. analyzed CT scans of 100 consecutive patients undergoing hip resurfacing arthroplasty surgery for hip osteoarthritis to find sex differences in hip morphology. In their study, the found no side-to-side difference for femoral antetorsion, femoral neck angle and femoral offset [26]. Using CT-based 3D femoral models, Dimitriou et al. compared the anatomy of 122 paired femora. Contrary to the findings of Atkinson 
et al., they demonstrated a statistically significant average side-difference in femoral antetorsion of $4.3^{\circ}$ (range $0^{\circ}-$ $\left.17.3^{\circ}\right)$. Similarly, the current study shows a mean sidedifference of $4.0^{\circ}$ (range $0.1^{\circ}-13.0^{\circ}$ ) for femoral antetorsion, although our findings were not statistically significant $(p=0.1)$. Together, these data suggest a high potential asymmetry of the femoral antetorsion, which should be considered whenever the contralateral femur is used as a reference for correction or restoration of an advantageous anatomy. Additionally, they showed a mean absolute side-difference of $2.5 \pm 2.1 \mathrm{~mm}$ for the horizontal offset, defined as the perpendicular distance between the anatomical femoral axis and the FHD, and $2.1 \mathrm{~mm} \pm 1.8 \mathrm{~mm}$ for vertical offset, which was defined as the vertical distance between a line connecting the tip of the lesser trochanter and the FHC. Although they did not measure the LTFHD, these findings indicate a potential side-difference of this parameter.

Although the current study demonstrated a statistically significant absolute side-difference of the average LTFH $\mathrm{D}$ of $1.6 \pm 1.4 \mathrm{~mm}$, this might not be of clinical relevance, since the resulting templating error for THA would be small and the majority of patients with minor LLD after THA have no or only few symptoms [2] However, osseous asymmetry might also occur in the pelvis. Therefore, during templating of total hip reconstruction, simple mirroring of the contralateral LTFHD without considering potential differences in pelvic morphology might lead to unsatisfiable results, since unrecognized concomitant femoral and pelvic asymmetry might cumulate and lead to symptomatic global leg length discrepancy. Furthermore, although side-differences in the LTFHD were not correlated with side-differences in femoral antetorsion, the intraoperatively measured LTFHD most likely depends on the torsion of the inserted femoral stem during THA. Consequently, alteration of the natural femoral antetorsion during THA might lead to variation of the intraarticular leg length compared to the preoperative templating. However, further studies are needed to quantify the impact of the torsion of the femoral stem on the intraoperatively measured LTFHD.

This study should be interpreted in light of its potential limitations. First, only limited demographic data were available of the examined specimens. Therefore, the impact of potential confounders such as ethnics and race could not be investigated. Second, only femora of subjects without any macroscopic pathology of the hip and the femur were included. Yet, the majority of patients requiring surgery suffer from altered anatomy due to degenerative or traumatic changes, which must be considered when THA is planned. Third, only femoral asymmetry was investigated in the current study. However, to achieve leg length equality, global leg length discrepancy and asymmetry of the pelvis must be taken into account, which was not assessed with the current study. Nonetheless, the current study provides the first 3D analysis of side-differences of the LTFHD. Therefore, our findings are free of projection errors resulting from measurements on plane radiographs, thus reflecting the intraoperative conditions more accurately. Furthermore, due to the standardized measurement method, the presented data demonstrated an excellent interclass ICC of 0.98 for measurement of the LTFHD. Lastly, no plane radiographs of the investigated specimens were available for analysis, thus a comparison of the 3D CT-based measurement of the LTFHD with measurements from 2D plane radiographs was not possible. Since measurements from plane radiographs are strongly influenced by the position of the patient (e.g. rotation/flexion of the hip), the findings from the current study might not necessarily correlate with measurements on plane radiograph. However, LTFHD is not only used for planning of total hip arthroplasty, but also for intraoperative validation of the transfer of the preoperative planning. The measurements of the LTFHD performed during surgery reflect the 3D CT-based measurements performed in this study. Thus, side-to-side difference, which remain unnoticed during preoperative planning on plane radiographs might lead to unintended Leg-Lengthdiscrepancy after total hip arthroplasty.

\section{Conclusions}

The LTFHD is a reliable reference parameter for preoperative templating and intraoperative validation during THA with only minimal side-to-side difference and a high correlation between sides $(\mathrm{r}=0.93, \mathrm{p}=<0.01)$. However, $8 \%$ of the current cohort showed a difference in LTFHD of more than $4 \mathrm{~mm}$, which should be anticipated during THA to avoid unsatisfiable results.

\section{Acknowledgements}

We thank the Institute of Forensic Medicine, University of Zürich, Switzerland for providing full-body - CT as well as demographic data of the investigated specimens.

\section{Authors' contributions}

$J H, A H$ und LV researched literature and conceived the study. $J H, A H, J A$ and $\mathrm{LV}$ were involved in protocol development and in data analysis. JH wrote the first draft of the manuscript. All authors reviewed and edited the manuscript and approved the final version of the manuscript.

\section{Funding}

There is no external funding source, or the funding source did not play a role in the investigation.

Availability of data and materials

The datasets generated and analysed during the current study are available from the corresponding author on reasonable request. 


\section{Declarations}

\section{Ethics approval}

Ethical approval for this study was obtained from Ethikkommission Zürich; ID $2017-01616)$

\section{Competing interests}

The authors declare no potential conflicts of interest with respect to the research, authorship, and/or publication of this article.

\section{Author details}

'Department of Orthopaedics, Balgrist University Hospital, University of Zurich, Forchstrasse 340, CH-8008 Zurich, Switzerland. ${ }^{2}$ Research in Orthopaedics Computer Science, Balgrist University Hospital, University of Zurich, Zurich, Switzerland.

Received: 6 December 2020 Accepted: 2 March 2021

Published online: 11 March 2021

\section{References}

1. Austin MS, Hozack WJ, Sharkey PF, Rothman RH. Stability and leg length equality in total hip arthroplasty. J Arthroplasty. 2003;18(3 Suppl 1):88-90.

2. Desai AS, Dramis A, Board TN. Leg length discrepancy after total hip arthroplasty: a review of literature. Curr Rev Musculoskelet Med. 2013;6(4): 336-41.

3. Meermans G, Malik A, Witt J, Haddad F. Preoperative radiographic assessment of limb-length discrepancy in total hip arthroplasty. Clin Orthop Relat Res. 2011;469(6):1677-82.

4. Scheerlinck T. Primary hip arthroplasty templating on standard radiographs. A stepwise approach. Acta orthopaedica Belgica. 2010;76(4):432-42.

5. Bono JV. Digital templating in total hip arthroplasty. J Bone Joint Surg Am. 2004;86-A Suppl 2:118-22.

6. Bourne RB, Rorabeck CH. Soft tissue balancing: the hip. J Arthroplasty. 2002; 17(4 Suppl 1):17-22

7. Clark CR, Huddleston HD, Schoch EP 3rd, Thomas BJ. Leg-length discrepancy after total hip arthroplasty. J Am Acad Orthop Surg. 2006;14(1): 38-45.

8. Matsuda K, Nakamura S, Matsushita T. A simple method to minimize limblength discrepancy after hip arthroplasty. Acta Orthop. 2006;77(3):375-9.

9. Gonzalez Della Valle A, Comba F, Taveras N, Salvati EA. The utility and precision of analogue and digital preoperative planning for total hip arthroplasty. Int Orthop. 2008;32(3):289-94.

10. Gonzalez Della Valle A, Slullitel G, Piccaluga F, Salvati EA. The precision and usefulness of preoperative planning for cemented and hybrid primary total hip arthroplasty. J Arthroplasty. 2005;20(1):51-8.

11. Kay RM, Jaki KA, Skaggs DL. The effect of femoral rotation on the projected femoral neck-shaft angle. J Pediatr Orthop. 2000;20(6):736-9.

12. Della Valle $A G$, Padgett $D E$, Salvati EA. Preoperative planning for primary total hip arthroplasty. J Am Acad Orthop Surg. 2005;13(7):455-62.

13. Suh KT, Kang JH, Roh HL, Moon KP, Kim HJ. True femoral anteversion during primary total hip arthroplasty: use of postoperative computed tomographybased sections. J Arthroplasty. 2006;21(4):599-605.

14. Unnanuntana A, Toogood P, Hart D, Cooperman D, Grant RE. The evaluation of two references for restoring proximal femoral anatomy during total hip arthroplasty. Clin Anat. 2010;23(3):312-8.

15. Sproul RC, Reynolds HM, Lotz JC, Ries MD. Relationship between femoral head size and distance to lesser trochanter. Clin Orthop Relat Res. 2007;461: $122-4$.

16. Sugano N, Noble PC, Kamaric E. Predicting the position of the femoral head center. J Arthroplasty. 1999;14(1):102-7.

17. Sariali E, Mauprivez R, Khiami F, Pascal-Mousselard H, Catonne Y. Accuracy of the preoperative planning for cementless total hip arthroplasty. A randomised comparison between three-dimensional computerised planning and conventional templating. Orthop Traumatol Surg Res. 2012; 98(2):151-8.

18. Lechler P, Frink M, Gulati A, Murray D, Renkawitz T, Bucking B, Ruchholtz S, Boese CK. The influence of hip rotation on femoral offset in plain radiographs. Acta Orthop. 2014;85(4):389-95.

19. Dimitriou D, Tsai TY, Yue B, Rubash HE, Kwon YM, Li G. Side-to-side variation in normal femoral morphology: 3D CT analysis of 122 femurs. Orthop Traumatol Surg Res. 2016;102(1):91-7.
20. Young EY, Gebhart J, Cooperman D, Ahn NU. Are the left and right proximal femurs symmetric? Clin Orthop Relat Res. 2013:471(5):1593-601.

21. Lindner C, Wallis GA, Cootes TF. Increasing shape modelling accuracy by adjusting for subject positioning: an application to the analysis of radiographic proximal femur symmetry using data from the Osteoarthritis Initiative. Bone. 2014;61:64-70.

22. Schneider P, Eberly D. Geometric Tools for Computer Graphics. Morgan Kaufmann Publishers Inc 2003:1056.

23. Jud L, Singh S, Tondelli T, Furnstahl P, Fucentese SF, Vlachopoulos L. Combined Correction of Tibial Torsion and Tibial Tuberosity-Trochlear Groove Distance by Supratuberositary Torsional Osteotomy of the Tibia. Am J Sports Med 2020:363546520929687.

24. Bonneau N, Libourel PA, Simonis C, Puymerail L, Baylac M, Tardieu C, Gagey $\mathrm{O}$. A three-dimensional axis for the study of femoral neck orientation. Journal of anatomy. 2012;221(5):465-76.

25. Polishchuk DL, Patrick DA Jr, Gvozdyev BV, Lee JH, Geller JA, Macaulay W. Predicting femoral head diameter and lesser trochanter to center of femoral head distance: a novel method of templating hip hemiarthroplasty. J Arthroplasty. 2013;28(9):1603-7.

26. Atkinson HD, Johal KS, Willis-Owen C, Zadow S, Oakeshott RD. Differences in hip morphology between the sexes in patients undergoing hip resurfacing. J Orthop Surg Res. 2010:5:76.

\section{Publisher's Note}

Springer Nature remains neutral with regard to jurisdictional claims in published maps and institutional affiliations.
Ready to submit your research? Choose BMC and benefit from:

- fast, convenient online submission

- thorough peer review by experienced researchers in your field

- rapid publication on acceptance

- support for research data, including large and complex data types

- gold Open Access which fosters wider collaboration and increased citations

- maximum visibility for your research: over $100 \mathrm{M}$ website views per year

At $\mathrm{BMC}$, research is always in progress.

Learn more biomedcentral.com/submission 